This item was submitted to Loughborough's Research Repository by the author.

Items in Figshare are protected by copyright, with all rights reserved, unless otherwise indicated.

\title{
Numerical analysis of thermo-mechanical behavior of indium micro-joint at cryogenic temperatures
}

PLEASE CITE THE PUBLISHED VERSION

http://dx.doi.org/10.1016/j.commatsci.2010.12.026

PUBLISHER

(C) Elsevier

VERSION

AM (Accepted Manuscript)

LICENCE

CC BY-NC-ND 4.0

\section{REPOSITORY RECORD}

Cheng, X., Changqing Liu, and Vadim V. Silberschmidt. 2019. "Numerical Analysis of Thermo-mechanical Behavior of Indium Micro-joint at Cryogenic Temperatures". figshare. https://hdl.handle.net/2134/13130. 
This item was submitted to Loughborough's Institutional Repository (https://dspace.lboro.ac.uk/) by the author and is made available under the following Creative Commons Licence conditions.

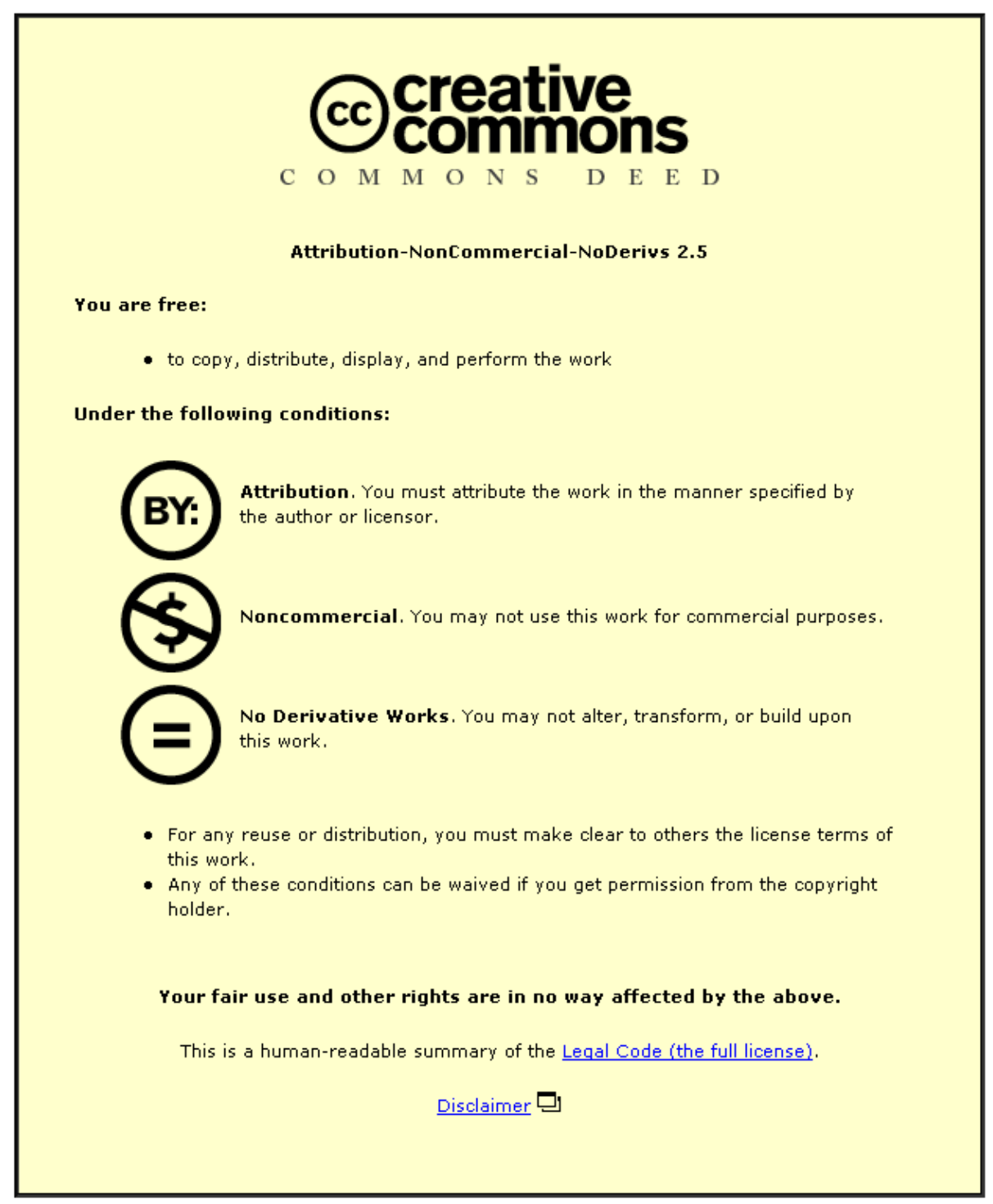

For the full text of this licence, please go to: http://creativecommons.org/licenses/by-nc-nd/2.5/ 


\title{
Numerical analysis of thermo-mechanical behavior of indium micro-joint at cryogenic temperatures X. Cheng. C. Liu, V.V. Silberschmidt
}

Wolfson School of Mechanical and Manufacturing Engineering, Loughborough University, Loughborough, Leicestershire, LE11 3TU, United Kingdom

\begin{abstract}
Microelectronic packaging plays an important role in cryogenic engineering; in particular, a solder joint as interconnection, which offers a mechanical, thermal and electrical support, undergoes much larger and harsher thermal changes during its service compared with conventional customer electronic products. The impact of thermo-mechanical properties of such solder joints under cryogenic service conditions becomes even more significant due to the continuing miniaturization of solder joints. Indium, a solder material with a low melting point and excellent cryogenic properties, has been favorable in various low temperature applications, in particular, to form solder joints for electronics interconnections. In order to understand the fundamental aspects of reliability of indium joints, this paper reports a constitutive model accounting for the effect of temperature change on thermo-mechanical behavior of indium joints. Especially, the model is used and subsequently implemented in a FE analysis to simulate a hybrid pixel detector system, in which indium micro-joints are manufactured to serve at cryogenic conditions. The response of indium joints to low-temperature cycling (300-76 K) was analyzed based on the proposed model, which not only offers a tool to understand the performance and experimental testing of solder joints under cryogenic temperatures, but can also be used for design optimization of the microelectronic package.
\end{abstract}

\section{Introduction}

Reliability of microelectronic packaging under changing thermal conditions is one of the main concerns for their design due to the mismatch of coefficients of thermal expansion (CTE) be- tween the different materials in the packages. This mismatch can induce residual stresses, resulting in thermal fatigue of a package under thermal loading due to service conditions or power cycling of devices.

Indium (In) is of considerable interest for interconnection in microelectronic packaging. 
In particular, indium bump bonding used in flip-chip assembly can offer high input/output numbers (I/Os), a fine pitch and small bump dimension $(<20 \mu \mathrm{m})$ [1-3]. The mismatch of CTEs between the substrate and connected chip, which is one of the most critical reliability issues in flip-chip assembly, can be accommodated due to indium's excellent ductility [4,5]. In addition, soldering with pure indium is considered to be a lowtemperature process due to the low melting-temperature of indium, which has been beneficial to a number of applications. A typical application is electronics integration for cryogenic engineering (e.g. satellites, spacecraft), for example, indium bump bonded interconnections in Josephson large-scale integrated (LSI) circuits [6] and in a hybrid pixel detector system. In such applications, the in-service thermal condition can be either far above the room temperature or down to cryogenic temperatures [7].

For a hybrid pixel detector system used in high-energy physics experiments, for example, European Organization for Nuclear Research (CERN), the service conditions require functions at cryogenic temperatures, i.e. below the temperature of liquid nitrogen $(76 \mathrm{~K})$. In such case, the temperature in the service is expected to change from cryogenic temperature to room temperature. It is also concerned that a relative impact of intrinsic properties of indium joints as interconnects in-service can become more significant as miniaturization requires micro-scale size of the joints.

In the present paper, the work is to investigate pure indium joints of $22 \mu \mathrm{m}$ diameter and $55 \mu \mathrm{m}$ pitch which are usually used to connect the sensor chip with the readout chip (for example, application specific integrated circuit (ASIC)) in Medipix 3 (a photoncounting pixel detector developed by CERN). The schematic structure of the package for Medipix 3 is shown in Fig. 1. The distribution and evolution of thermal stresses in indium micro-joints caused by temperature excursion from the room temperature (300 K) to

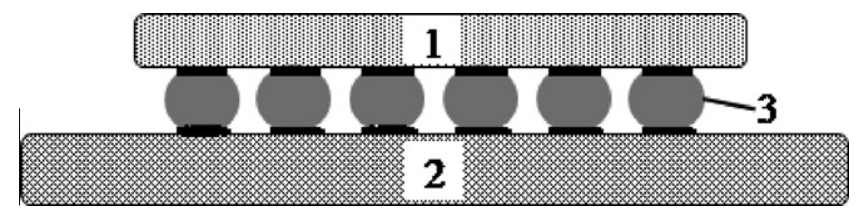

Fig. 1. Schematics of package for hybrid pixel detector system Medipix 3. 1, 2 and 3 stand for sensor, ASIC and indium bumps, respectively.

the liquid-nitrogen temperature (76 K) are investigated. A constitutive model to simulate these properties by considering this wide temperature change is therefore proposed and 
implemented into the finite-element analysis for numerical modeling.

\section{Finite element model}

\subsection{Model geometry}

Based on the Medipix 3 in Fig. 1, the geometry and structure of an FE model for one quarter of $6 \times 6$ array was built and simulated as this represents the part of the symmetric *package. The package configuration and the boundary conditions are illustrated in Fig. 2. Symmetric

boundary conditions are applied to both symmetric planes of the package (middle sections along axis $\mathrm{X}$ and $\mathrm{Z}$ ). Additionally, a displacement at the central bottom point of the readout chip was constrained along axis $\mathrm{Y}$ (marked in Fig. 2a). The FE mesh given in Fig. 2 was generated in ABAQUS with hexahedron element type (C3D8R). The under-bumps metallization between the sensor/readout chip and indium joint was neglected in order to simplify the model, thereby ideal bonding along their interfaces of three components were assumed.

\subsection{Constitutive model and material properties}

\subsubsection{Constitutive model}

Deformation mechanisms of materials depend on stress $\sigma$, strain-rate $\dot{\varepsilon}_{-}$, the homologous temperature $\mathrm{Th}$, which expresses the temperature of a material $(\mathrm{T})$ as a 
fraction of its melting-temperature $\left(\mathrm{T}_{\mathbf{m}}\right)$ using the Kelvin scale $\left(\mathrm{T}_{\mathbf{h}}=\mathrm{T} / \mathrm{T} \mathbf{m}\right)$, and their micro- structure (for example, grain size, impurities). Generally, the material's response under a given set of conditions can be presented by the flow law associated with more than one mechanism. For metals and alloys, the main mechanisms are plastic flow, powerlaw creep and diffusional flow as shown in Fig. 3. Plastic flow, which describes the time-independent plasticity, occurs at high-stress levels beyond the yield point. Powerlaw creep, which includes power-law breakdown, occurs at intermediate-stress level (10${ }^{4}<\sigma / \mathrm{E}<10^{-2}, \mathrm{E}$ is the Young's modulus). Diffusional flow, also called diffusional creep, occurs at low-stress region $\left(\sigma / \mathrm{E}<10^{-4}\right)$. Both power-law creep and diffusional flow are time- dependent. However, the range of dominance of each of the mechanisms is related not only to the stress, the strain-rate and the temperature, but also to the structure of the material. Taking the example of pure nickel, power-law creep can occurs around $0.2 \mathrm{~T} \mathrm{~m}$ for the grain size of $10 \mu \mathrm{m}$, where it could occurs around $0.1 \mathrm{~T} \mathbf{m}$ for a $1 \mathrm{~mm}$ grain size [8].

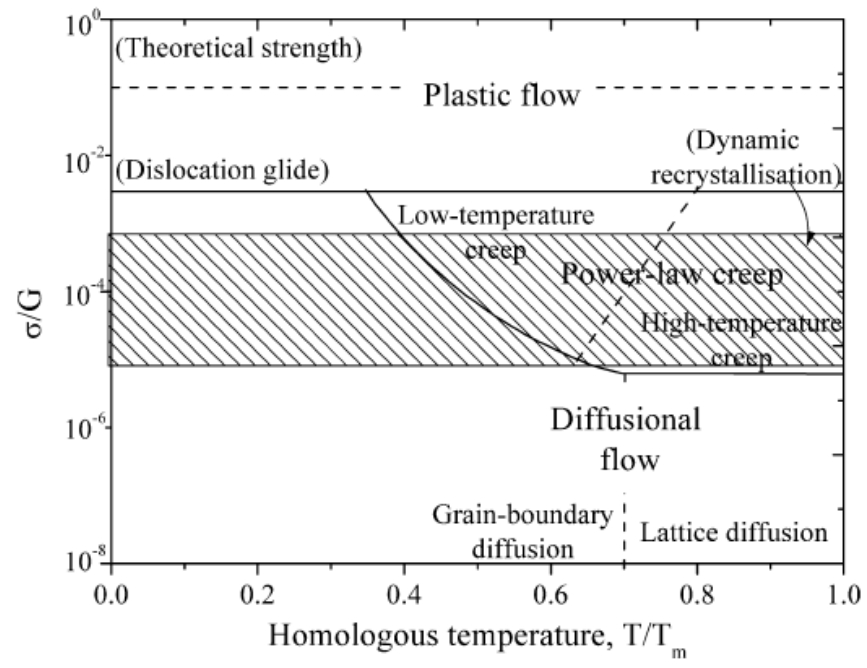

Fig. 3. Schematics of deformation mechanisms of metals and alloys: $G$ is shear

In electronics, residual stresses in solder joints are mainly resulted from the mismatch of CTEs of the components they inter connect. Under temperature excursions of the package in-service, the deformation/stress in a solder joint will depend on (i) temperature distribution as a function of time; (ii) parameters of the assembly; (iii) deformation performance of the solder. Considering the minimization of integrated circuits packages, temperature gradient in each package in-service is negligible (in our case, the overall dimension of the hybrid pixel detector package is less than 20 $\mathrm{mm}$ ). Therefore, the constitutive equation can be developed with the assumption of uniform temperature distribution.

Indium $(\mathrm{Tm}=429 \mathrm{~K})$, used as interconnection in hybrid pixel detector system, serves at cryogenic temperatures and undergoes temperature excursions over a wide range of homologous temperatures, for example, in this case, it may vary from $0.18 \mathrm{Tm}$ to $0.7 \mathrm{Tm}$ when temperature raises from $76 \mathrm{~K}$ to $300 \mathrm{~K}$. As stated above, the deformation of indium solder could be associated with the realisation of different mechanisms within this temperature regime. The total strain $\varepsilon_{\text {total }}$ can be defined as a sum of elastic strain $\varepsilon_{\mathrm{e}}$ and inelastic strain $\varepsilon_{\text {ie }}$ due to temperature excursions: 


$$
\varepsilon_{\text {total }}=\varepsilon_{e}+\varepsilon_{i e}
$$

For most metals and alloys, the experimental results have indicated that creep can be significant at temperatures above $0.3 \mathrm{~T}_{\mathrm{m}}$ [9]. So, the creep processes are expected to be the major inelastic deformation mechanism for this case. Referring to the general deformation map for pure metals as patterned in Fig. 3, thermal stress induced due to this temperature change could be described by power-law creep. However, over the wide temperature range of $0.18-0.7 \mathrm{~T}_{\mathrm{m}}$, the power-law breakdown is expected to occur to indium joint when the temperature decreases to approximately $0.5-0.6 \mathrm{~T}_{\mathrm{m}}$. The steady-state creep often prevails over primary or tertiary creep due to a relatively large fraction of creep life within this regime. And more general relationships were found to apply to steady-state creep of solder in a hyperbolic since form, which can account for different deformation mechanisms over a large range of stress and temperature via the same formula. Therefore, a hyperbolic sine function used to describe both the power-law and power-law breakdown was employed:

$$
\dot{\varepsilon}_{s s}=A \exp \left[-\frac{Q_{c}(T)}{R T}\right][\sin h \alpha(\sigma)]^{n}
$$

where $\mathrm{A}$ and a are constants, $\mathrm{R}$ is the gas constant, $\varepsilon_{\mathrm{ss}}$ is the steady-state creep strain-rate, $\mathrm{r}$ is the steadystate stress, Qc is the activation energy for creep, $\mathrm{T}$ is the absolute temperature and $\mathrm{n}$ is the stress exponent. It was found [10-12] that $\mathrm{Q}_{c}$ is essentially the activation energy for lattice self-diffusion $\mathrm{Q}_{s d}$ for a large class of materials over certain temperature range $\left(>0.6 \mathrm{~T}_{\mathrm{m}}\right)$. This is related to the deformation mechanism of materials which is temperature-dependent. Lattice-diffusion climbing is the major feature for materials above $0.6 \mathrm{~T}_{\mathrm{m}}$. However, the creep process is generally a thermally activated process, the level of $\mathrm{Q}_{\mathrm{c}}$ could change with the temperature, particularly when it is below $0.5 \mathrm{~T}_{\mathrm{m}}$. For a steady-state structure in the power-law regime, a constant modulus-compensated stress, termed as $\left(\frac{\sigma}{E}\right)$, approximately implies to be the equivalent stress under variable temperatures. A same equivalent stress can be referred to a fixed structure. Since the Young's modulus is the function of temperature, the temperature-dependent Young's modulus $(\mathrm{E}(\mathrm{T}))$ is considered to scale the steady-state stress in this case. Thus, for a given microstructure s and equivalent stress $\frac{\sigma}{E}$, the activation energy for creep $\mathrm{Q}_{\mathrm{c}}$ can be calculated as:

$$
Q_{c}=-\left.k\left[\frac{\partial\left(\ln \dot{\varepsilon}_{s S}\right)}{\partial(1 / T)}\right]\right|_{s=\text { constant }} ^{\frac{\sigma}{E}=\text { constant }}
$$


It is assumed that the constant level of $\left(\frac{\sigma}{E}\right)$ implies the constant structure. By adopting it (3), we obtained the value for activation energy at high temperature (defined as $\mathrm{Q}_{c}-\mathrm{HT}$ ) from the creep data for an indium joint under constant loads at $293 \mathrm{~K}, 343 \mathrm{~K}$ and $386 \mathrm{~K}$. Taking the concept of $\mathrm{Q}_{\mathrm{c}}$ declining with temperature [11,13-15], we selected the value of Qc at $76 \mathrm{~K}\left(\mathrm{Q}_{\mathrm{c}}-\mathrm{LT}\right)$ based on non-linear regression of the creep data at $76 \mathrm{~K}$ from Reed et al. [16] with (2). Then, a function of activation energy for creep with variable of temperature, $\mathrm{Qc}(\mathrm{T})$, was established as:

$$
Q_{c}(T)=\left\{\begin{array}{cc}
Q_{c}-H T, & T \geqslant 257.4 K \\
0.00046 T^{2}+0.15 T+10.34, & 76 K \leqslant T \leqslant 257.4 K \\
Q_{c}-L T, & T \leqslant 76 K
\end{array}\right.
$$

The non-linear regression of all creep data with (2) associating with $\mathrm{Q}_{c}(\mathrm{~T})$ was executed under the temperature range of 76-413 K. The constants required in (2) are listed in Table 1.

\section{Table 1}

Hyperbolic sine law creep model parameters obtained from regression of all creep data. Creep data: at 76 K [16]; at $173 \mathrm{~K}$ and $223 \mathrm{~K}$ [17]; 273-413 K [18]; 295-386 K from this study (unpublished).

\begin{tabular}{llll}
\hline$A\left(\mathrm{~s}^{-1}\right)$ & $\alpha\left(\mathrm{Pa}^{-1}\right)$ & $n$ & $Q_{c}(\mathrm{~kJ} / \mathrm{mol})$ \\
\hline $1.02 \times 10^{8}$ & $0.475 \times 10^{-6}$ & 5.86 & $Q_{c}(T)$ in $(4)$ \\
\hline
\end{tabular}

\subsubsection{Material properties}

The sensor and readout chips are both made of silicon which is input in the model. Since the indium joint is more ductile than silicon, the materials of the chips are assumed to be in the elastic state during the entire process. In this case, three types of properties are considered for the chips: the Young's modulus, Poisson's ratio and CTE. Apparently, the proposed constitutive model discussed above (defined as Case A) is applied to describe inelastic properties of indium joints outside the elastic range. In order to have a basis for comparison, a model with constant $\mathrm{Q}_{c}$ is also applied for indium joint as Case $\mathrm{B}$. As stated before, indium will undergo a wide range of homologous temperatures due to its low meltingtemperature; hence, the dependence of elastic and thermal properties on temperature has to be considered. CTEs of silicon in this study are also temperature-dependent so the effect of cryogenic temperatures on thermal stress can be included. The linear CTEs for silicon and indium as functions of temperature, implemented in the model, are given in Fig. 4 [19-24]. The global structure of the materials' properties applied to the model and other parameters used in the model are shown in Table 2.

\section{Table 2}

Material properties used in this study: the elastic properties of indium and silicon obtained from [25]. 


\begin{tabular}{|c|c|c|c|c|c|c|c|}
\hline & \multicolumn{3}{|c|}{ Elastic (300-76 K) } & \multirow[t]{2}{*}{ Expansion $(300-76 \mathrm{~K})$} & \multicolumn{3}{|c|}{ Creep (300-76 K) } \\
\hline & $E, \mathrm{GPa}$ & $v$ & Temperature, $\mathrm{K}$ & & & & \\
\hline \multirow[t]{3}{*}{ Indium } & 20.54 & 0.4326 & 76 & \multirow[t]{3}{*}{ CTE temperature-dependent (Fig. 4) } & \multirow{3}{*}{$\begin{array}{l}A, \alpha, n \text { in }(2) \\
\text { Table } 1\end{array}$} & $Q_{c}$ in $(2)$ & \\
\hline & 16.24 & 0.4408 & 187 & & & Case A & Case B \\
\hline & 12.70 & 0.4498 & 300 & & & $Q_{c}(T)$ in (4) & $Q_{\digamma}-H T$ \\
\hline Silicon & 16.80 & 0.28 & $76-300 \mathrm{~K}$ & CTE temperature-dependent (Fig. 4) & \multicolumn{3}{|l|}{$\mathrm{N} / \mathrm{A}$} \\
\hline
\end{tabular}

E - Young's modulus, v - Poisson's ratio, CTE - coefficient of thermal expansion

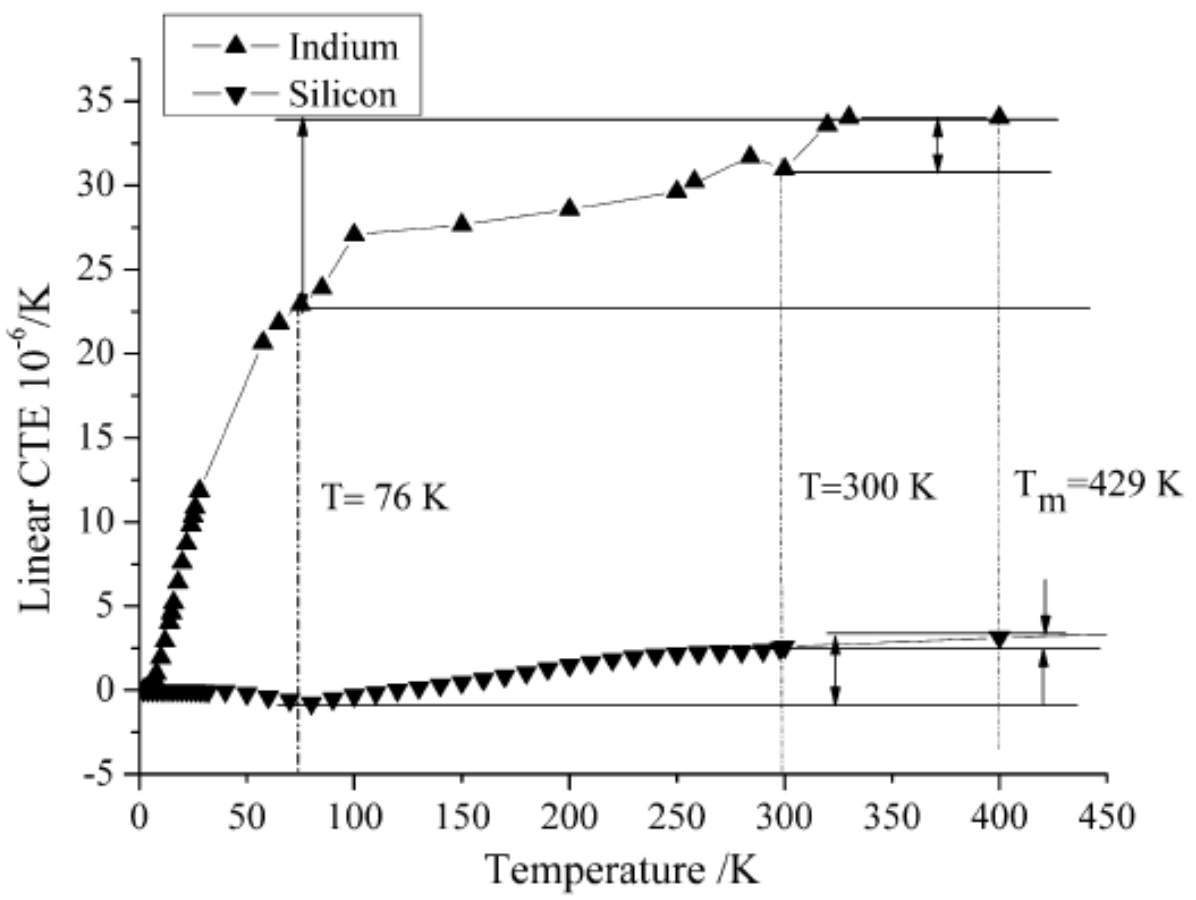

Fig. 4. Evolution of linear coefficient of thermal expansion of indium and silicon with temperature [1924]

\subsection{Thermal history}

The indium joint's response in the package to five low-temperature cycles was simulated. Since the package dimensions are small, the temperature gradient is neglected in this study. The temperature is assumed to be homogenous in the package, and it changes with the ambient temperature. The heating and cooling rate of a thermal cycle is $2.5 \mathrm{~K} / \mathrm{s}$; with the temperature range being from $300 \mathrm{~K}$ to $76 \mathrm{~K}$ and the dwell time of $80 \mathrm{~s}$. The temperature change during low-temperature cycling is illustrated in Fig. 5.

\section{Results and discussion}

\subsection{Case A}

\subsubsection{Stress distribution under low-temperature cycling}

The distribution of von-Mises stress in the package after five low-temperature cycles is presented in Fig. 6, which clearly shows that the corner joint at the edges of the package (arrowed in Fig. 6) is the main site of stress concentration due to low-temperature cycling. Although both chips are made of silicon, different 
displacements are induced in them due to the displacement constraint along axis $\mathrm{Y}$ at the central bottom point of the readout chip (marked in Fig. 2a) and dissimilarity of their dimensions. Consequently, it results in stress concentration and deformation of indium joints.

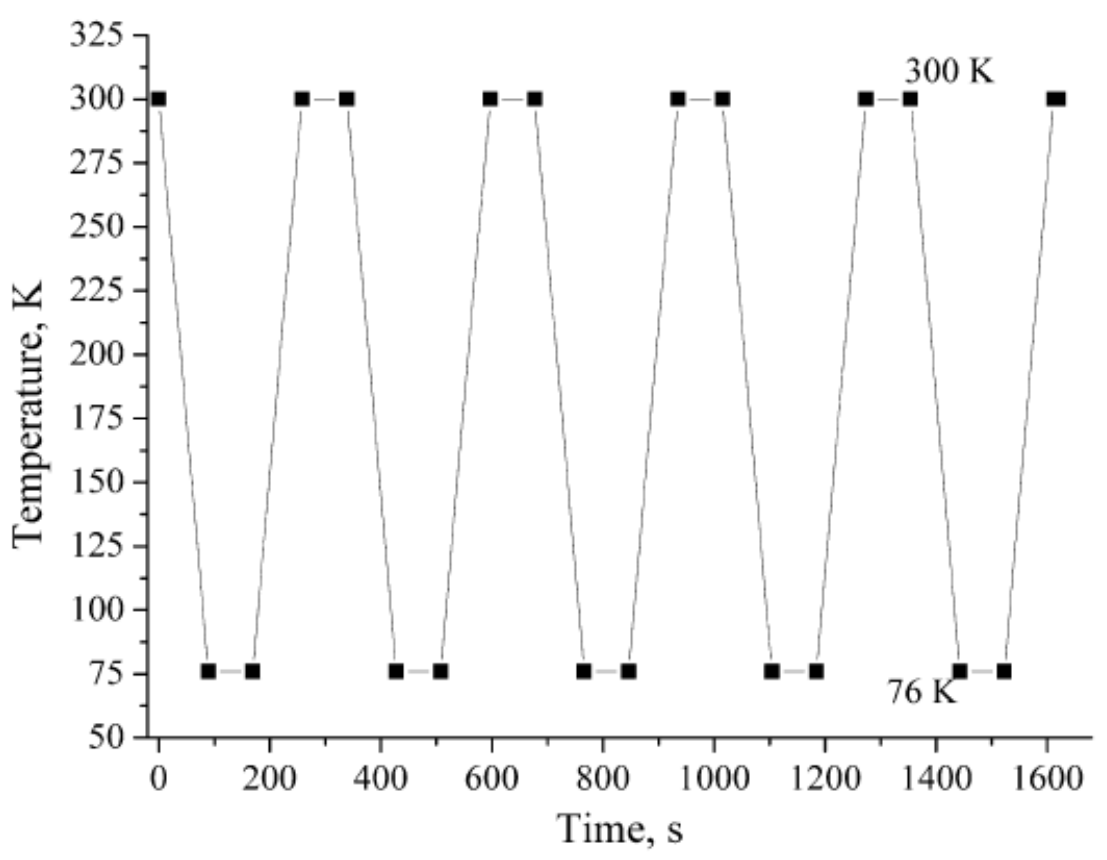

Fig. 5. Profile for low-temperature cycling used in this study

Since displacements of the sensor and readout chips at their edges are larger than that at the centre; the peripheral joints will be subject to more serious deformation, resulting in larger residual stresses. For a single joint, stresses are concentrated at the solder/chip interfaces as seen in the outmost joint (Fig. 7). Also, the stress level in the central zone near the interface of the solder/ readout chip in Fig. 7 is higher than that near the solder/sensor chip interface. Because the readout chip is normally assembled with a multi-chip module (MCM) through solder bumps, the sensor chip has more degrees of freedom for stress relaxation than the readout chip. Above all, under a low-temperature cycling load, the interfaces of the outmost joint are one of the most critical positions in the package.

\subsubsection{Effect of temperature on stress distribution and its evolution}

In order to understand the influence of temperature change on stress concentration, a von-Mises stress distribution in the outmost joint is evaluated when the temperature decreases to $76 \mathrm{~K}$ (Fig. 8a) and then increases to $300 \mathrm{~K}$ (Fig. 8c). As can be seen, the deformed shape obtained after cooling process shown in Fig. 8a demonstrates that the indium joint mainly underwent a tensile loading as temperature decreased. And the peripheral region of the joint has the highest residual stresses during temperature changes. To study this distribution, all stress components were analyzed at two locations at the solder/sensor interface (Points 1 and 2 in Fig. 8a). 


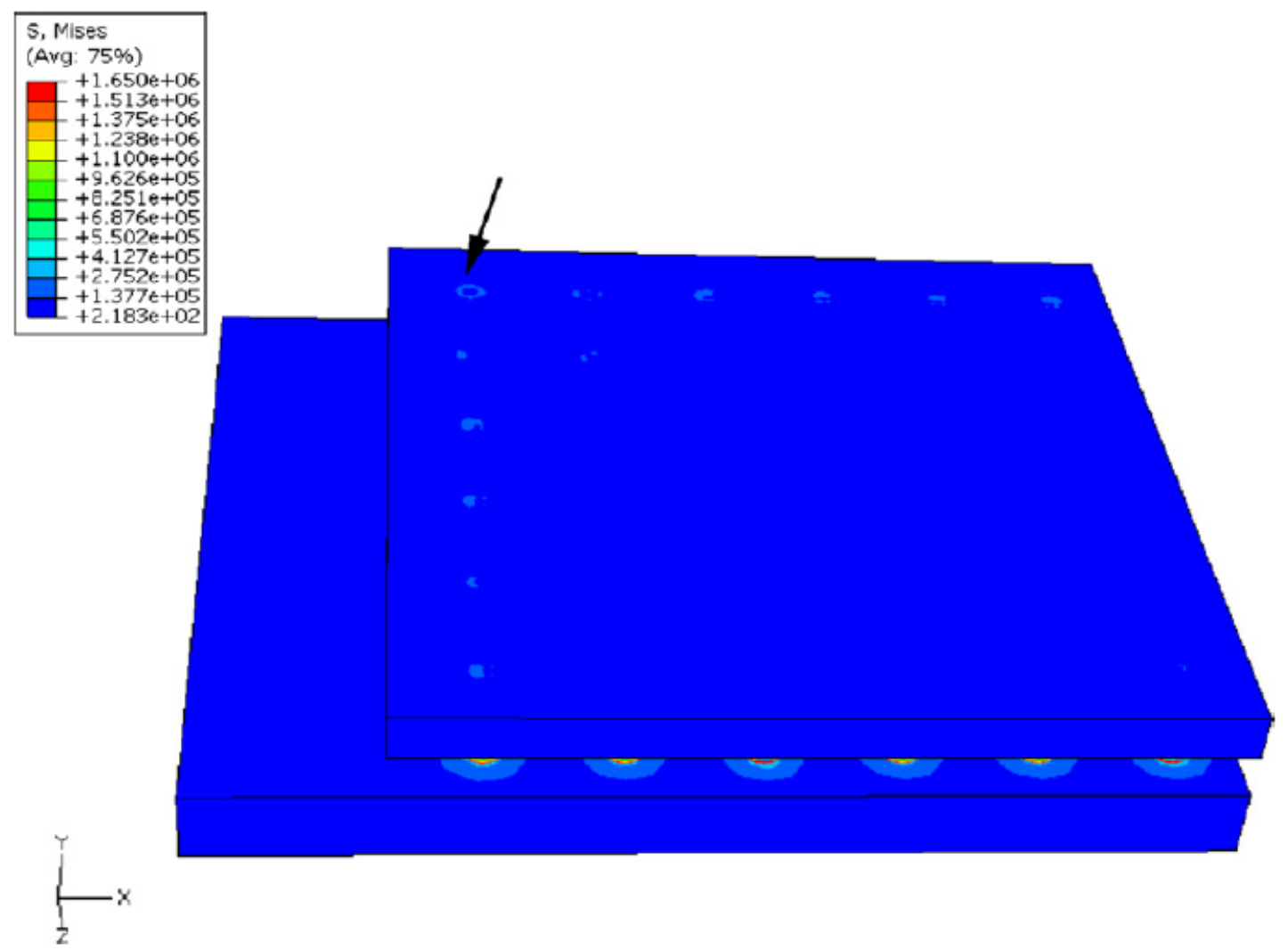

Fig. 6. Distribution of von-Mises stress in package after five low-temperature cycles

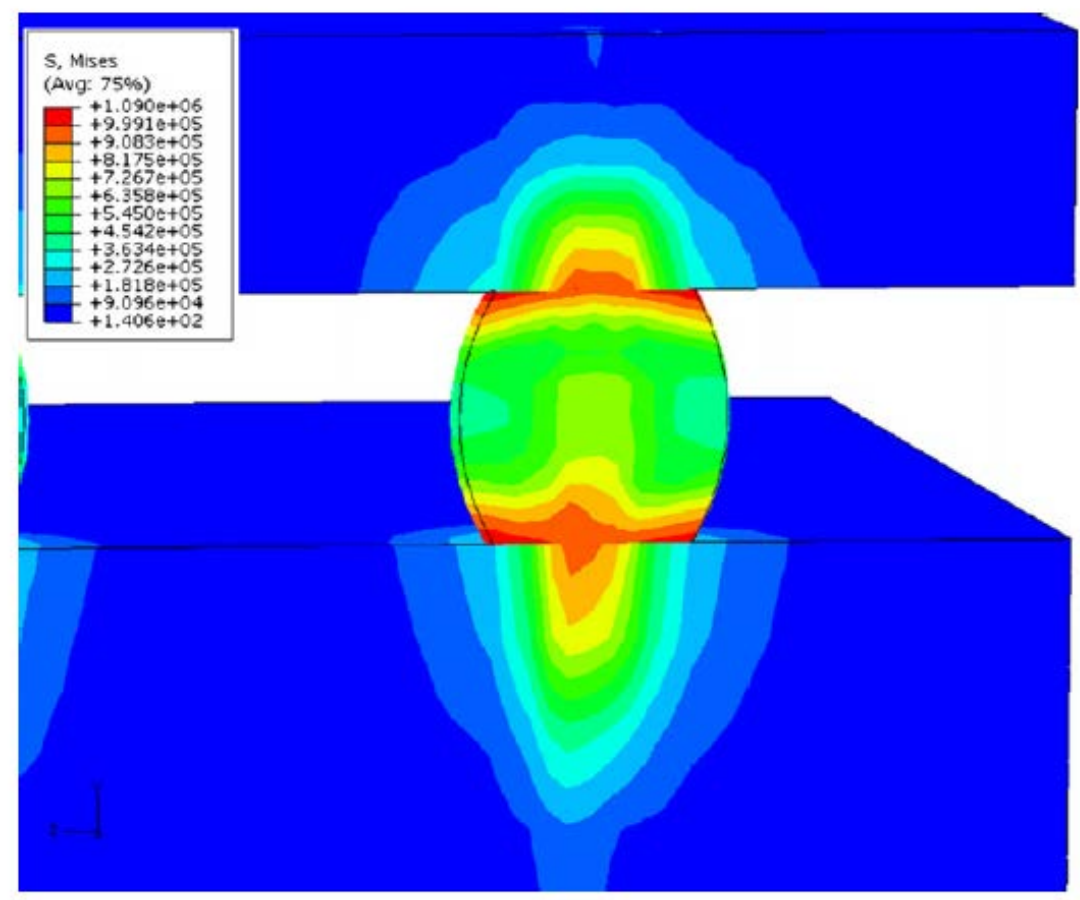

Fig. 7. Distribution of von-Mises stress in outmost joint (in its middle cross-section) after five lowtemperature cycles 
It was found that the largest components are the normal stresses $\sigma_{11}, \sigma_{22}$ and $\sigma_{33}$, the levels of which are in the same order of magnitude with the von-Mises stress and an order higher than that of other components. Focusing on Points 1 and 2, $\sigma_{11}$ and $\sigma_{33}$ are tensile for both locations; by contrast, the value of $\sigma_{22}$ is positive at Point 2 and negative at Point 1, indicating that these two points underwent tension and compression with the decrease of temperature (Fig. 8b). Hence, the inverse distribution in $\sigma_{22}$ should be the major source of stress concentration in Fig. 8a, according to the von-Mises criteria.

(a)

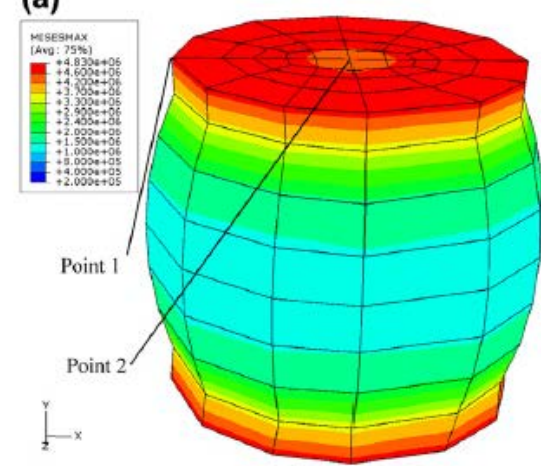

(c)

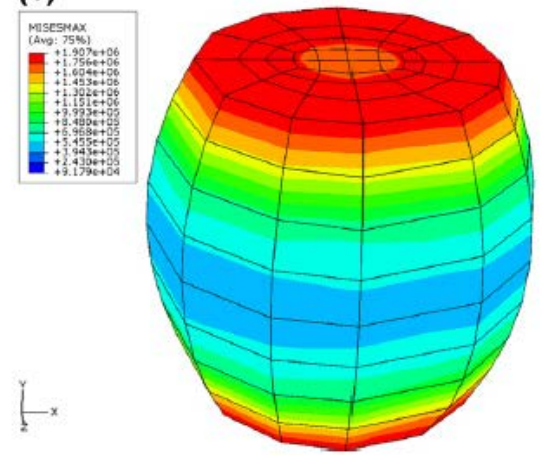

(b)

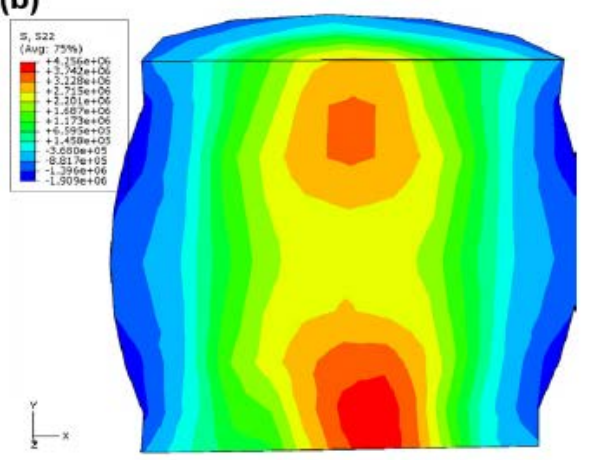

(d)

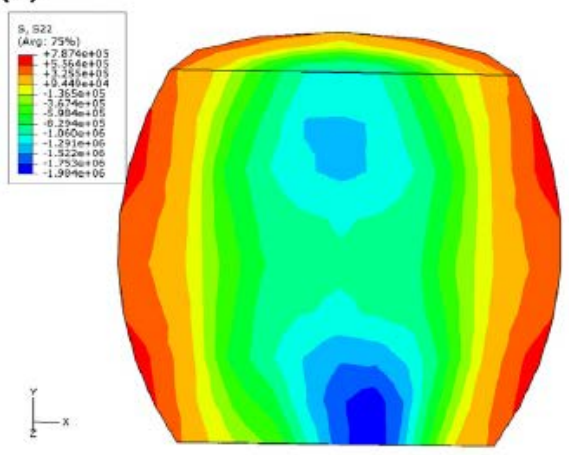

Fig. 8. Stress distribution in outmost joint: (a) von-Mises stress and (b) stress component $\sigma_{22}$ (central cross-section) when temperature is reduced to $76 \mathrm{~K}$ ( $\mathrm{t}=89.2 \mathrm{~s}$ ); (c) von-Mises stress and (d) stress component $\sigma_{22}$ (central cross-section) when temperature is increased to $300 \mathrm{~K}(\mathrm{t}=258.4 \mathrm{~s}$ ). Points 1 and 2 are locations of the outmost joint for comparative study. Scale factor for deformation is 20.

By assuming isotropic thermal expansion, the level of contraction of indium is higher in all the directions compared to silicon during a cooling process since the CTE of indium is much larger than that of silicon (Fig. 4). Thus, the distance between the sensor and the readout chips (Y axis) decreased as a result of a large amount of contraction of indium joint. Meanwhile, for a single joint, the interfaces between it and chips restrained the contraction of indium along $\mathrm{Y}$ axis due to the mismatch in CTEs of indium and silicon, which resulted in an inverse distribution in $\sigma_{22}$ between the edge and central regions at the solder/chip interfaces. Subsequently, the edge region near the solder/chip interfaces exhibited the largest deformation due to the highest level of residual stress induced when the temperature decreased to $76 \mathrm{~K}$ as 
seen in Fig. 8a. Nevertheless, the additional constraint on geometry (fixed displacement along Y axis at the central bottom point in Fig. 2a) contributed to the appreciable difference in stress distribution $\left(\sigma_{22}\right)$ between the indium/sensor (top-plane) and indium/readout (bottom-plane) interfaces shown in Fig. 8b. Still, no high shear deformations were observed in the indium joint during low-temperature cycling, which could be linked to the different level of contraction mismatch between sensor/readout chips and solder/ chips. Shear deformation is normally related to the mismatch between top layer (sensor) and bottom layer (readout chip). In our case, since both chips are made of silicon, the source for this mismatch is mainly due to the limited geometry constraint. According to our results, the contraction mismatch caused by the differential CTEs between the solder and chips is likely to be predominant, leading to tension/compression deformation, rather than shear deformation due to the geometry constraint.

It is similar to the case when temperature increases from $76 \mathrm{~K}$ to $300 \mathrm{~K}$ as given in Fig. 8d, which is attributed to the differential expansion of indium and silicon associated with constraints due to the interfaces of indium joint. Consequently, the edge near the solder/chip interface appeared to be the major site for deformation during the temperature change (for instance, the heating and cooling process).

The influence of temperature on the stress evolution was also analyzed for two stages: the dwell period at cryogenic temperature (down-dwell stage) and at the maximum temperature (up-dwell stage), respectively. Point 1 (Fig. 8a) at the solder/sensor interface was analyzed since it is the main site for stress concentration and deformation. The signs ( 4 ) and ( $)$ in Fig. 9a represent the von- Mises evolution of Point 1 after normalization during down- and up-dwell stages, respectively. Stress relaxation occurred in both down- and up-dwell stages. Approximately, 37\% of stresses were released during the down-dwell stage while more than $65 \%$ relaxation occurred in the up-dwell stage as expected since material hardens at cryogenic temperature. Analysis of creep deformation at Point 1 demonstrates that the creep strain increased with time at both stages as obvious in Fig. 9b, but the amount of the increase during the downdwell stage ( 4 ) is larger than that during the up-dwell stage ( $\bullet$ ). This is attributed to the change of creep strain with time with the creep strain rate depending on stress and the value of $\left(\mathrm{Q}_{c} / \mathrm{T}\right)$ according to (2). Thus, the larger increase at cryogenic level could be related to the fact that residual stress at low temperature is higher than at maximum temperature as shown in Fig. 8a and c, and the activated energy for creep applied to cryogenic temperature $\left(\mathrm{Q}_{c}-\mathrm{LT}\right)$ is much lower than at maximum temperature $\left(\mathrm{Q}_{\mathrm{c}}-\right.$ HT).

\subsubsection{Deformation at low-temperature cycling}

Evolution of the effective inelastic strain $\left(\varepsilon_{\text {eff }}^{i . e .}\right)$ with low-temperature cycles was obtained with (5) on the basis of the von-Mises criteria and plotted in Fig. 10.

$$
\varepsilon_{\text {eff }}^{\text {i.e. }}=\frac{\sqrt{2\left[\left(\varepsilon_{1}^{\text {i.e. }}-\varepsilon_{2}^{\text {i.e. }}\right)^{2}+\left(\varepsilon_{2}^{\text {i.e. }}-\varepsilon_{3}^{\text {i.e. }}\right)^{2}+\left(\varepsilon_{3}^{\text {i.e. }}-\varepsilon_{1}^{\text {i.e. }}\right)^{2}\right.}}{3}
$$



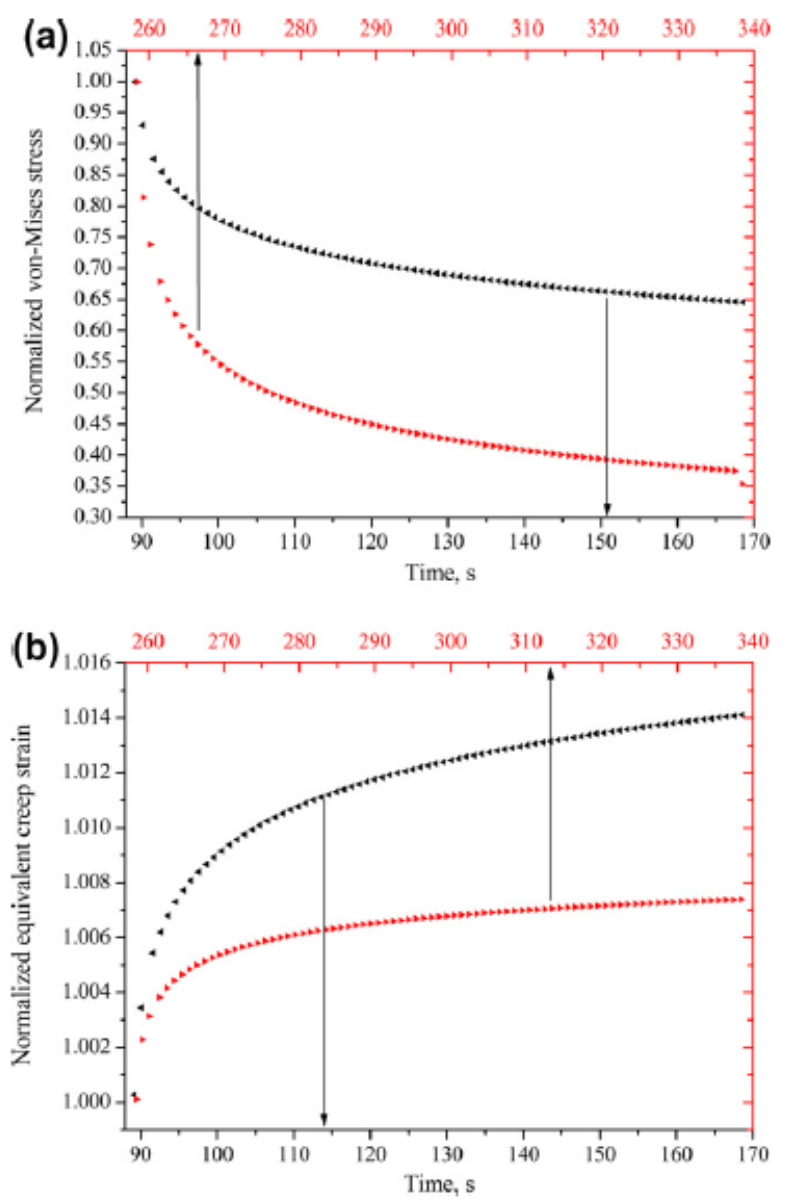

Fig. 9. Evolution of von-Mises stress (a) and equivalent creep strain (b) at Point 1 (see Fig. 8a): when temperature is reduced to $76 \mathrm{~K}(\mathrm{t}=89.2 \mathrm{~s})$ and dwelled for $80 \mathrm{~s}$ during first cycle (bottom coordinate axis); when temperature is increased to $300 \mathrm{~K}(\mathrm{t}=258.4 \mathrm{~s}$ ) and dwelled for $80 \mathrm{~s}$ during first cycle (top coordinate axis). Signs ( 4 ) and ( ) represent the respective down- and up-dwell stages.

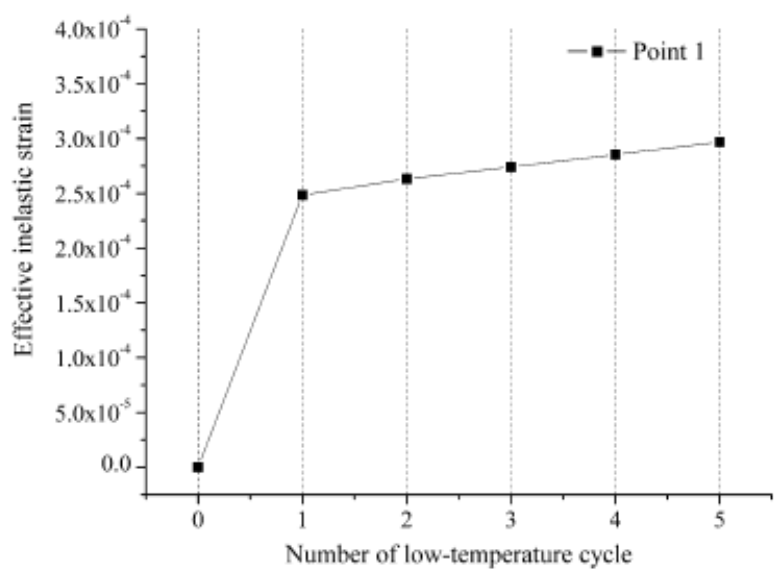

Fig. 10. Evolution of effective inelastic strain at Point 1 with thermal cycles 
where $\varepsilon_{i}^{i . e .}(\mathrm{k}=1-3)$ is the principal inelastic strain component. Thermal ratcheting occurred in the joint caused by low-temperature cycling as seen in Fig. 10 (Point 1). And the stabilized inelastic strain tends to occur after one cycle.

\subsection{Case B}

In a general numerical procedure to analyze the reliability of solder joints due to temperature changes in electronics packages, the solder's response in a package under conditions of thermal cycling test is simulated with the FE model. The constitutive law applied to describe the creep behavior of solder joints is commonly attained by fitting the experimental creep data obtained from the tests with a series of constant loads at evaluated temperatures. As stated before, the creep behavior of metals and alloys for normal service conditions usually can be expressed in terms of a powerlaw relation, including stress, creep strain (rate), and temperature and activation energy for creep. Here, the curve-fitting process was simplified to execute the relation by assuming a constant activation energy for creep within the range of temperature studied. It is valid for temperature regimes above $0.5 \mathrm{~T}_{\mathrm{m}}$ of the solder material. For our case, we adopted this concept as Case $\mathrm{B}$, by employing $\mathrm{Q}_{\mathrm{c}}-\mathrm{HT}$ (the value corresponding to temperature above $0.6 \mathrm{~T}_{\mathrm{m}}$ shown in (4) as a constant level of activation energy during the low-temperature cycling and compared it with Case A in order to understand the influence of activation energy on the deformation of solder joints due to low-temperature cycling.

Evolution of von-Mises stress during two stages (down- and up-dwell stages) at Point 1 was compared and plotted after normalization in Fig. 11a. Compared with stress development, the capability of stress relaxation predicted in Case A is 35\% in average, which is larger than that in Case B (5\% relaxation) during the down-dwell stage (bottom coordinate axis in Fig. 11a) while approximately 65\% residual stress induced during heating process was released for both Cases A and B during the up-dwell stage (top coordinate axis in Fig. 11a). Fig. 11b illustrates the equivalent creep strain of both Cases A and B during the down-dwell stage after normalization. Apparently, a larger increase is found in Case A compared with Case B. Combined with the comparison of creep strain evolution between the down- and up-dwell stages in Case A (Fig. 9b), the of the outmost joint (i.e. Point 1), which is significantly higher than the yield strength (2.8 MPa), and the ultimate strength (15 MPa), of pure indium at $76 \mathrm{~K}$ [26], becoming unrealistic in field use. By contrast, the stress magnitude predicted in Case A is about 4.83 MPa at Point 1 after cooling the package down to $76 \mathrm{~K}$ with a cooling rate of $2.5 \mathrm{~K} / \mathrm{s}$ (Fig. 8a). For a period of dwell at $76 \mathrm{~K}$ for 80 s, Fig. 9a clearly indicates the 35\% maximum stress was relaxed (down to $3.14 \mathrm{MPa}$ ) at Point 1. This is reasonable since the fast cooling rate will induce higher residual stress (the rate of $2.5 \mathrm{~K} / \mathrm{s}$ used in our study is much faster than that encountered in-service).

The difference in the creep deformation at Point 1 between Case A and Case B is presented for five lowtemperature cycles in Fig. 12. The predicted value for Case A is $27.3 \%$ higher than that for Case B. Refocusing on the creep increase along thermal cycling: as expected as a result of the differential level of 
creep deformation during the down-dwell stage, there is a higher increase (16\%) in creep strain during the down-dwell stage for Case A as given in Fig. 11b.
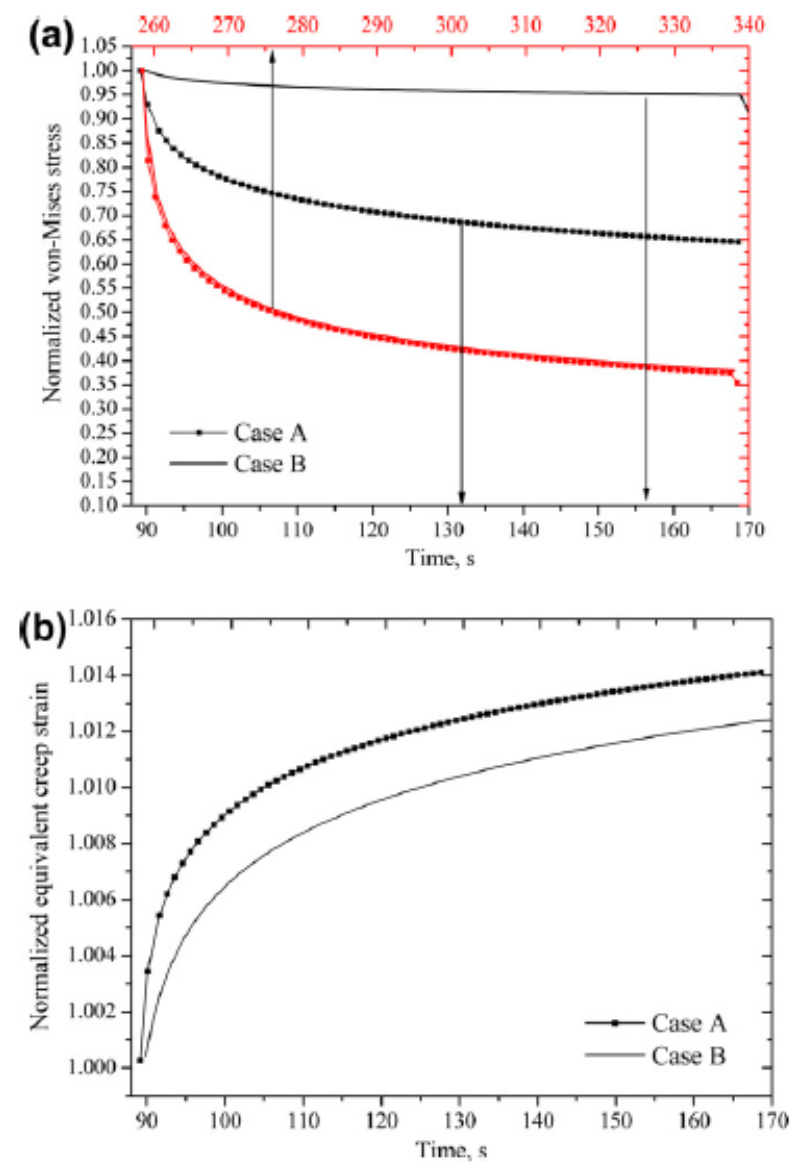

Fig. 11. Evolution of von-Mises stress and equivalent creep strain at Point 1 (see Fig. 8a): (a) von-Mises stress during down-dwell stage (bottom coordinate axis) and up-dwell stage (top coordinate axis); (b) equivalent creep strain during down-dwell stage (from t = 89.2 to $169.2 \mathrm{~s}$ ): Signs (--) and ( ) stand for down- and up-dwell stages of Case A; Signs (-) and ( ) stand for down- and up-dwell stages of Case B.

\section{Conclusions}

In this paper, a constitutive model, accounting for the influence of the large-range temperature change $\left(0.2-0.7 \mathrm{~T}_{\mathrm{m}}\right.$ of a material) on the deformation, is proposed for the indium joints used in cryogenic applications, specifically associated with the system of hybrid pixel detector, thereby, the solder's response in the package to low-temperature cycling (76-300 K) was studied.

The results show that the peripheral regions of the solder/chip interfaces are the critical locations that can be likely induce failure, in particular, for the outmost joints. Thermal ratcheting occurred to the joints during low-temperature cycling. For the two cases studied, compared to the results from the Case B based on a constant level of the activation energy for creep, the proposed approach (Case A) can produce more adequate results for the temperature change. 


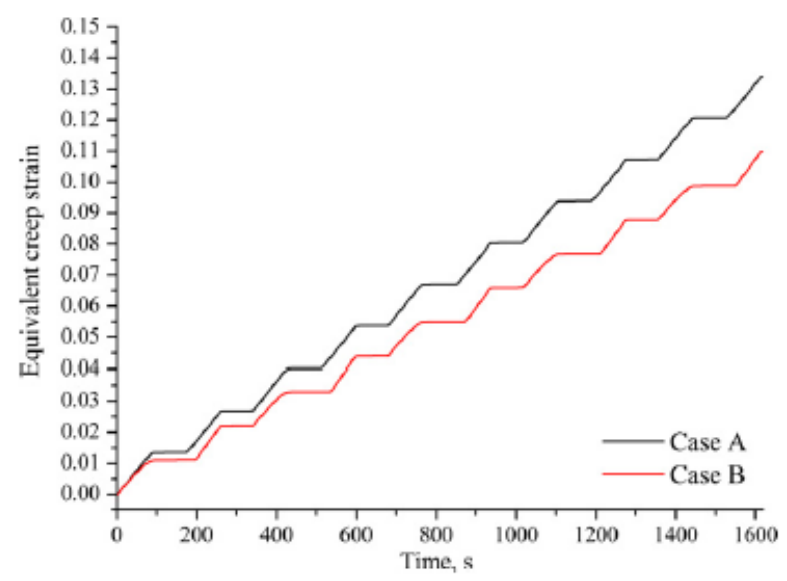

Fig. 12. Evolution of equivalent creep strain at Point 1 (Fig. 8a) with low-temperature cycles.

The effect of variable activation creep energy should be accounted in the thermo-mechanical stress and deformation analysis of a device used at cryogenic temperatures as it is likely to increase the sensitivity of creep strain-rate to low temperature. The proposed model offers an approach to analyze the performance of the joints under temperature change of concerns, and provides a useful tool for design optimization for the electronic package and experimental testing. The future research will consider the comparison between the obtained experimental data from indium joints due to the thermal cycling between $76 \mathrm{~K}$ and $300 \mathrm{~K}$.

\section{References}

[1] S. Kwan, J. Andresen, J.A. Appel, G. Cardoso, Study of indium and solder bumps for the BTeV pixel detector, in: Nuclear Science Symposium Conference Record IEEE, New York, USA, 2003, p. 59.

[2] J. John, L. Zimmermann, P.D. Moor, C.V. Hoof, Nuclear Instruments and Methods in Physics Research Section A: Accelerators, Spectrometers, Detectors and Associated Equipment 531 (1-2) (2004) 202-208.

[3] M. Lozano, E. Cabruja, A. Collado, J. Santander, M. Ullán, Nuclear Instruments and Methods in Physics Research Section A: Accelerators, Spectrometers, Detectors and Associated Equipment 473 (1-2) (2001) 95-101.

[4] M. Plötner, G. Sadowski, S. Rzepka, G. Blasek, Microelectronics International 8 (2) (1991) 27-30.

[5] G. Humpston, D.M. Jacobson, Advanced Materials and Processes 163 (4) (2005) 45-47.

[6] J. Yeh, Metallurgical and Materials Transactions A 13 (9) (1982) 1547-1562. [7]

B.A. Hands (Ed.), Cryogenic Engineering, first ed., Academic Press, Harcourt 
Brace Jovanovich, London, 1986.

[8] H.J. Frost, Deformation-Mechanism Maps: The Plasticity and Creep of Metals and Ceramics, first ed., Pergamon, Oxford, UK, 1982.

[9] M.E. Kassner, Introduction in Fundamentals of Creep in Metals and Alloys, second ed., Elsevier, Amsterdam, London, 2009. pp. 1-8.

[10] S.L. Robinson, O.D. Sherby, Acta Metallurgica 17 (2) (1969) 109-125.

[11] H. Luthy, A.K. Miller, O.D. Sherby, Acta Metallurgica 28 (2) (1980) 169-178.

[12] R. Darveaux, Mechanical Evaluation of Indium for Die Attachment in a

Multichip Package, North Carolina State University, 1993, pp. 82-83.

[13] R.E. Frenkel, O.D. Sherby, J.E. Dorn, Acta Metallurgica 3 (5) (1955) 470-472.

[14] O.A. Ruano, J. Wadsworth, O.D. Sherby, Acta Metallurgica 36 (4) (1988) 11171128.

[15] J. Weertman, Journal of Applied Physics 28 (10) (1957) 1185-1189.

[16] R.P. Reed, R.P. Walsh, Advanced in Cryogenic Engineering (Materials) 38 (1992) 117-126.

[17] W. Rui, F.P. McCluskey, Journal of Electronic Materials 38 (9) (2008) 18551859.

[18] J. Weertman, Transaction of the American Institute of Mining and

Metallurgical Engineers 218 (2) (1960) 207-218.

[19] J.G. Collins, J.A. Cowan, G.K. White, Cryogenics 7 (1-49) (1967) 219-224. [20]

O. Prakash, R. Ashok, P.N. Dheer, Journal of Physics 39 (6) (1992) 655-660. [21] J.F.

Smith, V.L. Schneider, Journal of the Less-Common Metals 7 (1964) 17-22. [22] P.W. Sparks, C.A. Swenson, Physical Review 163 (3) (1967) 779-790.

[23] G.K. White, Journal of Physics D: Applied Physics 6 (1973) 2070-2078. [24]

W.M. Yim, R.J. Raff, Journal of Applied Physics 45 (3) (1974) 1456-1457.

[25] R. Darveaux, Mechanical Evaluation of Indium for Die Attachment in a

Multichip Package, PhD Thesis, North Carolina State University, 1993.

[26] R.P. Reed, C.N. McCowan, R.P. Walsh, L.A. Delgado, J.D. McColskey, Materials

Science and Engineering A 102 (1988) 227-236. 